\title{
Las guerrillas en el Ejército Popular de la República (1936-1939)
}

\author{
Hernán Rodríguez Velasco \\ Doctor en Historia por la Universidad de Salamanca \\ hernanhistoria@gmail.com
}

Recibido: 10/12/2009

Aceptado: 24/02/2011

\section{RESUMEN}

Utilizando documentación primaria e inédita, el autor repasa cronológicamente las medidas que el alto mando republicano llevó a cabo durante la Guerra Civil Española para encuadrar las espontáneas prácticas de los grupos de guerrilleros bajo la ortodoxia del Ejército Popular. Por primera vez en la historiografía se analiza este proceso de organización, atendiendo además a su funcionamiento interno y a las contradicciones y problemas que se produjeron con el resto de unidades regulares.

Palabras clave: Guerra Civil Española, guerrillas, Ejército Popular de la República, XIV Cuerpo de Ejército, S.I.A.M., Manuel Estrada, Vicente Rojo.

The Guerrillas in the Popular Army of the Spanish Republic(1936-1939)

\begin{abstract}
By using original and previously unknown records, the author chronologically describes the measures taken by the Republican Military Staff during the Spanish Civil War that brought the guerrilla fighters' spontaneous actions of the under the Popular Army's orthodoxy. It is the first time in the historiography that this organization process has been assessed, focusing on the internal operations as well as the contradictions and problems related to the rest of the Army's units.
\end{abstract}

Key words: Spanish Civil War, guerrillas, Popular Army of the Republic, XIV Cuerpo de Ejército, S.I.A.M., Manuel Estrada, Vicente Rojo.

Sumario: Introducción. 1. Breve apunte sobre las guerrillas y los ejércitos. 2. Huidos y guerrilleros (julio-diciembre 1936). 3. Disciplinar la espontaneidad (enero-junio 1937). 4. Composición, entrenamiento y algunas quejas (junio-septiembre 1937). 5. Creación y desarrollo del SIAM o XIV Cuerpo de Ejército (octubre 1937-febrero 1938). 6. Misiones, eficacia y problemas de mando (marzo-diciembre 1938). 7. Conclusiones.

\section{Introducción}

Sorprende comprobar cómo la Guerra Civil Española, uno de los conflictos armados más estudiados por la historiografía mundial, tiene aún lagunas por cubrir. Desde el punto de vista estrictamente bélico las ausencias son bastante significativas y, en con- 
creto, la historia militar ha reparado más bien poco en torno al fenómeno de las guerrillas republicanas ${ }^{1}$. La mayoría de los libros sobre grupos partisanos se ha centrado fundamentalmente en el período posbélico, cuando los famosos maquis continuaron la resistencia contra el régimen franquista hasta casi principios de los años sesenta. Esta bibliografía específica se ha nutrido de estudios generales, investigaciones locales o regionales e incluso con testimonios personales ${ }^{2}$.

Sin embargo, para el período comprendido entre 1936 y 1939 no existe hasta la fecha ningún estudio monográfico sobre este aspecto, y las menciones a las partidas de guerrilleros han sido bastante breves o tangenciales. Destacan por su interés las obras de Eduardo Pons Prades y Secundino Serrano, que abarcan los años del conflicto mediante una aproximación a la figura de los huidos, guerrilleros espontáneos que posteriormente fueron encuadrados o no en el Ejército Popular ${ }^{3}$.

El problema de estos libros es que no inciden sobre la inclusión de estas guerrillas o la formación de unas nuevas dentro de la organización militar republicana durante la Guerra Civil. De ello se ocuparon, ya desde finales de los setenta, Domingo Pastor Petit y Michael Alpert ${ }^{4}$. Sus aportaciones abrieron un camino para conocer algo sobre esta forma irregular de lucha, si bien estos autores utilizaron para ello exclusivamente documentación del enemigo, basada muchas veces en informes contradictorios de desertores o prisioneros. Asimismo, su mención a dos importantes hechos, el famoso decreto de Rojo en diciembre de 1936 y la creación del XIV CE a mediados de 1937, no aparecen conectados de manera lógica, y el resto de la información que aportan dibuja un panorama muy general con pinceladas de anécdotas sobre su modo de actuación.

El presente artículo trata de las guerrillas que formaron parte del Ejército Popular. En las siguiente líneas se pretende revisar algunas ideas y fechas erróneas que se han asumido como ciertas, descifrar el proceso de regulación de las guerrillas desde antes de diciembre de 1936 y más allá de mediados de 1937, aportar más datos sobre su funcionamiento interno, su adscripción orgánica en la ortodoxia militar y los problemas que surgieron entre ellas y otras unidades regulares. Con el fin de profundizar en el análisis del fenómeno guerrillero se partirá de una contextualización del

${ }^{1}$ Sobre las lagunas en la historia militar en este período vid. RODRÍGUEZ VELASCO, Hernán: "La historia militar y la Guerra Civil Española: una aproximación crtítica a sus fuentes" Studia Historica. Historia Contemporánea., Vol. 24, 2006, pp. 57-80

${ }^{2}$ El número de obras es cada vez mayor. Destacamos aquí sólo unas pocas: ARÓSTEGUI, Julio y MARCO, Jorge (eds.): El último frente. La resistencia armada antifranquista en España 1939-1952, Madrid, Catarata, 2008; MORENO GÓMEZ, Francisco: La resistencia armada contra Franco. Tragedia del maquis y la guerrilla, Barcelona, Crítica, 2002; MARCO, Jorge: "Guerrilla, bandolerismo social, acción colectiva. Algunas reflexiones metodológicas sobre la resistencia armada antifranquista" en Cuadernos de Historia Contemporánea, 2006, vol. 28, pp. 281-301; YUSTA, Mercedes: Guerrilla armada y resistencia campesina: la resistencia armada contra el franquismo en Aragón (1939-1952), Zaragoza, Universidad de Zaragoza, 2003.

${ }^{3}$ PONS PRADES, Eduardo: Guerrillas españolas 1936-1960, Barcelona, Planeta, 1977 y SERRANO, Secundino: Maquis. Historia de la guerrilla antifranquista, Madrid, Temas de Hoy, 2001, pp. 23-67.

${ }^{4}$ ALPERT, Michael: El Ejército Popular de la República, 1936-1939, Barcelona, Crítica, 2007 (3 $3^{\text {a }}$ edición), pp. 301-307 y PASTOR PETIT, Domingo: Los Dossiers Secretos de la Guerra Civil, Barcelona, Argos 1978, pp. 143-152. 
mismo a nivel global, para después reflexionar sobre su aparición en el proceso de militarización del bando leal y cuestionar el apoyo o desinterés que recibió por parte de los mandos, militares y políticos, republicanos. Desde el punto de vista metodológico, y como novedad, el estudio se apoya en documentación inédita que además es de origen republicano, y que contradice las palabras de Alpert cuando afirma que la documentación conservada sobre las guerrillas procede "generalmente de fuentes nacionales, lo cual quiere decir que en el Ministerio republicano de Defensa Nacional o bien no se creaban documentos al respecto o bien se hicieron desaparecer al final de la guerra, y puede deberse a que las autoridades republicanas no las controlaban totalmente"

\section{Breve apunte sobre las guerrillas y los ejércitos}

El uso de las guerrillas o pequeños grupos armados que combaten contra un ejército regular es bastante antiguo. Las acciones rápidas y sorpresivas de Indíbil y Mardonio o Viriato en Hispania, o de otros insurgentes en Judea contra la dominación romana dan cuenta de ello. Lo que es más raro es la colaboración de estos grupos con unidades regulares. En España, las partidas de Espoz y Mina, El Empecinado o Porlier en la lucha contra Napoleón inauguraron en 1808 una tradición que, no muchos años más tarde, continuaría el general Zumalacárregui en las guerras carlistas. Su inferioridad numérica le hizo recurrir a componentes guerrilleros en algunas batallas.

Este fenómeno ya había sido recogido por el teórico de la guerra Carl von Clausewitz, para quien "los campesinos armados debían complementar el propio ejército permanente del país y deberían unirse a él de vez en cuando para atacar guarniciones y hostigar conntingentes mayores de las fuerzas de ocupación" al tiempo que el ejército regular "debería inspirar y ayudar a organizar a los campesinos armados".

Sin embargo, este tipo de lucha no cuajó en la mayoría de las guerras decimonónicas. Sólo fue algo recurrente a finales de siglo cuando las potencias europeas se enfrentaron a pueblos africanos o asiáticos en sus guerras de dominación colonial. La asimetría de los contendientes llevó a hablar de small wars, que también "incluían la guerra de guerrillas que normalmente surge cuando soldados entrenados son empleados para sofocar sediciones e insurrecciones en los países civilizados"7.

Durante la Gran Guerra, el uso de armas de repetición que causaban grandes masacres a la infantería enemiga provocó un orden táctico más abierto para disminuir su vulnerabilidad, aunque no sería hasta la Segunda Guerra Mundial cuando se normalizaron procedimientos de infiltración de unidades tipo sección (30-50 hombres) y pelotón (10-20 hombres) en las líneas enemigas con el fin de sabotear sus acciones o destruir sus infraestructuras. Las guerrillas, o mejor dicho, grupos regulares usando técnicas guerrilleras comenzó sólo entonces a ser algo común en los ejércitos del

${ }^{5}$ ALPERT, M.: El Ejército..., pp. 303-304

${ }^{6}$ HEUSER, Beatrice: Reading Clausewitz, London, Pimlico, 2002, p. 137

${ }^{7}$ CALLWELL, C.E.: Small Wars: Their Principles and Practice, London, HMSO, 1906 cit. en FREEDMAN, Lawrence (ed.): War, Oxford, Oxford University Press, 1994, p. 315 
mundo. Precisamente, la actuación de las guerrillas dentro del Ejército Popular en la Guerra Civil Española inspiró a muchos excombatientes internacionales para luchar contra la ocupación nazi o fascista en la URSS, Francia, Italia, Grecia o Yugoslavia entre 1939 y 1945 . De hecho, al frente de los grupos del Ejército de Liberación de Yugoslavia estuvieron cuatro comandantes que habían combatido en las Brigadas Internacionales; Irving Goff, William Aalto y Vince Lossowski, viejos componentes de la Brigada Lincoln, pertenecieron después al Office of Strategic Services norteamericano que entrenó a españoles para operar detrás de las líneas alemanas en el Norte de África en 1943 y que en Nápoles contactó con partisanos italianos para coordinar su lucha en el norte del país; en la URSS, a la dinamitera Elizaveta Parshina la NKVD soviética le encomendó en 1942 preparar un grupo terrorista cuya misión consistiría en la realizar actos de sabotaje en Moscú en caso de que la ciudad fuera entregada a los alemanes, el coronel general Mámsurov fue uno de los jefes de la Dirección General de Inteligencia Militar del EM de las Fuerzas Armadas soviéticas e Iliá Grigórievich Stárinov fue instructor y director de escuelas guerrilleras en Rusia, Ucrania y Polonia ${ }^{8}$.

Tras la Segunda Guerra Mundial, la Guerra Civil Griega (1946-1949) siguió desarrollándose por parte de uno de los bandos en clave guerrillera, y este tipo de lucha se reprodujo al calor de las guerras revolucionarias y anticoloniales o de liberación. La amenaza nuclear inhibió una forma de lucha convencional y las prácticas de agitación y sabotaje triunfaron en la China maoísta, en la Cuba del Che y en el Vietnam del general Giap. En este último país las técnicas guerrilleras y la actuación del ejército regular se asimilaron de manera total. En la actualidad, los conflictos más recientes exigen un nuevo modo de luchar y, de hecho, "las nuevas estrategias bélicas aprovechan la experiencia tanto de la guerrillas como de la lucha contrarrevolucionaria" con el fin de "evitar el combate y hacerse con el territorio a través del control político de la población"9. De modo que, a pesar del paso del tiempo, el uso de métodos guerrilleros sigue siendo hoy, como hace cientos de años, una forma eficaz de enfrentarse al enemigo.

${ }^{8}$ CRUSELLS, Magí: Las Brigadas Internacionales en la pantalla, Ciudad Real, Universidad de Castilla la Mancha, 2002, p. 109; CARROLL, Peter N: The Odyssey of the Abraham Lincoln Brigade: Americans in the Spanish Civil War, Standford, Standford University Press, 1994, pp. 270-273; GERASSI, John: The Premature Antifascists. North American Volunteers in the Spanish Civil War, 1936-1939, An Oral History, New York, Praeger, 1967, p. 214; PARSHINA, Elizaveta: La brigadista. Diario de una dinamitera en la guerra civil, Madrid, La Esfera de los Libros, 2002, p. 348; RYBALKIN, Yuri: Stalin y España. La ayuda militar soviética a la República. Madrid: Marcial Pons, 2007, p. 114

9 KALDOR, Mary: Las nuevas guerras. Violencia organizada en la era global, Barcelona, Tusquets, 2001, pp. 22 y 23 


\section{Huidos y guerrilleros (julio-diciembre 1936) ${ }^{10}$}

Cuando comenzó la Guerra Civil Española, los actos de represión cometidos por los sublevados empujaron a muchos ciudadanos de pueblos y ciudades a huir o a esconderse. Este fenómeno no era nuevo ya que en 1919, 1931 o 1934 "en momentos de especial acoso represivo, militantes destacados en el obrerismo optaron por quitarse de en medio, abandonar los pueblos y refugiarse en el campo, hasta que pasase la escabechina"11. La novedad de 1936 es que esta vez ya no pudieron volver a sus casas. Algunos de ellos quedaron aislados en zonas dominadas desde el principio por el enemigo (Galicia, León, Huelva, Granada), lo que hizo casi imposible cualquier contacto con la zona leal. Todo el Norte peninsular también quedaría incomunicado desde octubre de 1937 con las sucesivas caídas de Vizcaya, Santander y Asturias ${ }^{12}$. Estos huidos eran personajes que resistían, que luchaban fundamentalmente por su propia supervivencia y que, por tanto, "estaban a la defensiva". Sus acciones se limitaban "a vengarse de las represalias, con el fin de asustar al enemigo e impedir que cometieran otras. Se trataba más de sobrevivir que de iniciar una ofensiva"13. La mayoría de ellos fueron perseguidos y ejecutados durante la guerra ${ }^{14}$.

Los guerrilleros, es decir, huidos más organizados y que habitaban en zonas colindantes o muy próximas a la España republicana (Aragón, Extremadura, sector SurTajo), no se limitaron solamente a resistir, sino que, desde un principio, también fomentaron voladuras de puentes, de vagones de trenes o de vehículos y agitaron a la población local distribuyendo propaganda por dichas zonas ${ }^{15}$. Es el caso de las partidas de Ramón Rufat y otros guerrilleros de la zona a los que el coronel republicano José Eduardo Villalba, responsable del frente de Huesca en aquellos momentos, había confiado la lucha militar en determinados sectores de dicho frente. La porosidad de estos lugares facilitaba la infiltración de partisanos como los del Grupo Machuca, cuyas acciones están documentadas por el Ministerio de la Guerra en octubre de $1936^{16}$.

${ }^{10}$ He querido redefinir aquí los conceptos que distingue Francisco Moreno para la etapa posbélica. Este autor utiliza el término huido para el período 1939-1944 “en el que predominó el carácter fugitivo ante el terror franquista" y guerrillero se aplica a la etapa organizada del fenómeno con una apariencia de estructura militar y jerárquica en MORENO, F.: La resistencia ... p. 1.

${ }^{11}$ MORENO, F.: La resistencia... p. 4.

12 Un ejemplo de esto en MARTÍNEZ-LÓPEZ "EL QUICO”, Francisco: Guerrillero contra Franco. La Guerrilla antifranquista de León (1936-1951), León, Diputación de León, 2000, p. 38.

13 FRASER, Ronald: Recuérdalo tú y recuérdalo a otros. Historia oral de la Guerra Civil Española, II. Barcelona: Crítica, 1979, pp. 177-178. La "resistencia pasiva" en Asturias en García Piñeiro, Ramón: Los mineros asturianos bajo el franquismo (1937-1962), Madrid, Fundación Primero de Mayo, 1990.

14 SOLÉ I SABATÉ, Josep y VILLARROYA, Joan: "La represión de los huidos y la guerrilla en la zona sublevada" en Juliá díAz, Santos (coord.): Víctimas de la guerra civil, Madrid: Temas de Hoy, 1999, pp. 187-202.

15 Para el caso de las guerrillas extremeñas vid. CHAVES PALACIOS, Julián: Huidos y maquis. La actividad guerrillera en la provincia de Cáceres (1936-1950), Diputación Provincial, Cáceres, 1994 y VILA IZQUIERDO, Justo: La guerrillas antifranquista en Extremadura, Universitas Editorial, Badajoz, 1986.

16 RUFAT, Ramón: Espions de la République. Mémoires d'un agent secret pendant la guerre 
Junto a ellos, las escasas columnas de regulares que actuaban en el perímetro madrileño habían sido autorizadas también por el Estado Mayor del Ministerio para que, "previa la aprobación del Mando", pudieran "proponer y ejecutar los golpes de mano que considere necesarios para completar sus informes"17. Es decir, que desde muy temprana fecha el Estado permitió la actuación de grupos guerrilleros con fines informativos al tiempo que seguía muy de cerca las actuaciones de otros grupos espontáneos en los que presumiblemente delegaba parte de su responsabilidad defensiva.

Las causas de esto último deben buscarse en el decreto del presidente José Giral licenciando a las tropas bajo la autoridad de los militares sublevados y que, paradójicamente, provocó la espantada de las unidades regulares del bando leal ${ }^{18}$. Las milicias de partidos y sindicatos y grupos de corte guerrillero en general trataron en aquellos primeros momentos de sustituir al Ejército aunque con escaso éxito. De ahí que el Gobierno de Largo Caballero iniciara a partir de septiembre la reconstrucción del nuevo Ejército Popular bajo nuevos parámetros ideológicos y logísticos, cuya célula principal sería la Brigada Mixta y donde se imponía la militarización de las milicias. El Partido Comunista a través del ejemplo del Quinto Regimiento fue el principal impulsor y valedor de dichas reformas. Asimismo, en octubre, la llegada de voluntarios extranjeros determinó la creación de las primeras Brigadas Internacionales. En apenas un trimestre las fuerzas republicanas lograron reconstruir el Ejército, aunque con la lógica reticencia de anarquistas y algunos grupos nacionalistas ${ }^{19}$.

De esta forma, cuando comenzó la batalla de Madrid en el mes de noviembre, los mandos militares republicanos pudieron contar con unidades regulares que defendieron la capital, mientras aún coleaban grupos armados por encuadrar. Al término de dicha batalla, el Jefe del Estado Mayor de la Junta de Defensa de Madrid, el coronel Vicente Rojo, valoró la calidad de algunos de estos grupos irregulares y "ordenó la formación de unidades guerrilleras a partir de la XII Brigada Internacional y el Quinto Regimiento. Consistirían en dos grupos de 50 hombres" ${ }^{20}$.

Paradójicamente, este decreto asumía la creación de un servicio "con la finalidad de disponer de fuerzas organizadas capaces de actuar de una manera irregular", cuya misión era "el hostigamiento sobre la retaguardia enemiga, realizando golpes de mano en profundidad sobre las comunicaciones, convoyes, Centros de abastecimiento, puestos de Mando etc., y lo efectuarán de una manera metódica por sorpresa y en relación con los planes que preparará o se halle desarrollando el mando"21.

d'Espagne. Paris: Éditions Allia, 1990, pp. 20 passim y Centro Documental de la Memoria Histórica (en adelante CDMH), INCORPORADOS, 739, Cp. 104 y CDMH, INCORPORADOS, 737, Cp. 122, Exp. 4.

${ }^{17}$ Archivo General Militar de Ávila (en adelante AGMAV), C. 227, Cp. 6, D. 4/2 (12 agosto de 1936). Por golpes de mano se entendía acciones rápidas de tipo guerrillero. La cursiva es nuestra.

${ }^{18}$ Gaceta de Madrid, Núm. 201, 19 de julio de 1936, p. 274

${ }^{19}$ El proceso de militariación en ENGEL, Carlos: Historia de las Brigadas Mixtas del Ejército Popular de la República 1936-1939, Madrid, Almena, 2005, pp. 5-9.

${ }^{20}$ ALPERT, M.: El Ejército..., pp. 302-303.

${ }^{21}$ AGMAV, C. 788, Cp. 21, D. 2 / 1. 


\section{Disciplinar la espontaneidad (enero-junio 1937)}

Rojo había demostrado que confiaba en las guerrillas. El otro militar que apostaba con igual o más fe que él por estos grupos era el Jefe del Servicio de Información del Estado Mayor del Ministerio, Manuel Estrada. Ya en agosto, había permitido los golpes de mano, y de nuevo ahora, resaltaba que las guerrillas "no son ni pueden ser una organización regular militar propiamente dicha; pero forman un elemento auxiliar de valor inapreciable, de que el mando debe servirse y que conviene incrementar cuanto sea posible en todos los frentes" 22 . Por esta razón se trató de promover mejoras para que pudiesen trabajar en condiciones más óptimas y ofreciesen un mayor rendimiento: se pensó en editar un reglamento sobre la labor a realizar por los guerrilleros, en centralizar su dirección para evitar la demora en las informaciones, en dotarlas con mejor armamento e incluso en designar un crédito para subvencionar las tareas más arriesgadas ${ }^{23}$.

A comienzo de año, las guerrillas extremeñas acababan de cosechar varios éxitos con la voladura del ferrocarril entre Talavera y Madrid. Esto motivó la preparación del famoso Plan Extremadura, que pretendía aislar Andalucía del resto de España y obligar al enemigo a descongestionar el frente Norte y el cerco de Madrid, mediante la conquista del nudo de comunicaciones de Mérida y la ocupación de la región de Oropesa $a^{24}$.

Aunque el plan sufriría diversas cancelaciones y no se llevaría a cabo hasta muchos meses más tarde, los altos mandos republicanos, con el beneplácito del Presidente del Gobierno, pergeñaron hacia mediados de enero de 1937 el entonces Plan para información, destrucciones y levantamiento en masa de la región de Extremadura. Con él se pretendía no sólo ejercer actos de sabotaje, sino también conseguir un gran levantamiento en masa de la población extremeña donde "existen numerosísimos partidarios de nuestra causa y se dan condiciones, como el malestar económico y el terror fascista, cuya explotación puede beneficiarnos extraordinariamente por crearnos el ambiente propicio" ${ }^{25}$. El plan iba a ser dirigido por dos rusos, el teniente coronel Santi en colaboración con el coronel Daivis. Para ello las guerrillas se considerarían desde entonces "como [una] unidad más del Ejército de la República siquiera funcione con las características de irregularidad y relativa independencia que exige su cometido"26.

Como vemos, desde las altas esferas republicanas se promovía la creación y la participación de las guerrillas como partes del engranaje militar, lo que contradice las palabras del consejero ruso Stepanov cuando afirma que la organización de mo-

\footnotetext{
${ }^{22}$ CDMH, INCORPORADOS, 737, Cp. 69, Exp. 7.

${ }^{23}$ Ibídem.

${ }^{24}$ El estudio más detallado sobre el Plan Extremadura en CAMPANARIO, Juan Miguel: "Los proyectos fallidos del Ejército Popular de la República para dividir en dos la zona ocupada por el enemigo: el Plan P del general Vicente Rojo" en http://www2.uah.es/jmc/planp.pdf.

${ }^{25} \mathrm{CDMH}$, INCORPORADOS, 675, Cp. 20.

${ }^{26}$ Ibídem.
} 
vimientos guerrilleros "fue realizada a espaldas del propio Caballero" 27 . De hecho, éste aprobaría en enero de 1937 un proyecto para explotar la labor de estos grupos disponiendo para ello de nuevos cuadros de combatientes: "hacia el 8 de febrero organizar dos escuelas para la preparación de gente. En Valencia para 15-20 personas y en Madrid para 10-15 personas" ${ }^{28}$.

Pero Largo Caballero no era el único que apoyaba la creación de guerrillas. Si algún partido estuvo a favor de fomentar esta práctica, ése fue el Partido Comunista. Contrariamente a lo que afirma Secundino Serrano de que "existía una manifiesta hostilidad del PCE y una parte del Partido Socialista Obrero Español (PSOE) hacia una hipotética formación de unidades guerrilleras" 29 , comunistas destacados como Enrique Líster, José Díaz, Vicente Uribe o Carlos García se postularon a favor de la lucha guerrillera ${ }^{30}$. No sabemos si por propia convicción o por presión soviética. Está claro que desde Moscú, se apostó por esa vía como lo demuestra la conversación entre el consejero militar Yan Karlovich Berzin con el guerrillero Artur Sprogis para empezar en febrero de 1937 "la Guerra de los raíles"

Hasta el 15 de marzo la actividad guerrillera se desarrolló a lo largo de cuatro sectores (Extremadura, Jaén, Málaga y Aragón), pero en ese momento se dio por concluido "el período de ensayo de las guerrillas para comenzar la fase de desarrollo hasta el logro de una organización de tipo verdaderamente nacional, pudiendo convertirse en verdaderas compañías de choque [...] en este momento se inicia la creación de guerrillas del Ejército del Norte, una vez constituidas éstas, serán cinco las agrupaciones que actuarán: Jaén, Extremadura, Madrid, Aragón y Norte"32.

Un mes más tarde llegaba el informe donde se notificaban los trabajos a realizar y la estructura del "trabajo especial" entre el 15 de marzo y el 15 de abril de 1937. Para entonces ya se habían configurado hasta siete sectores más de actuación con un jefe cada uno: Extremadura (el más desarrollado ya que tenía subsectores), Monterrubio de la Serena, frente Sur, Talavera de la Reina (este grupo se ocupaba especialmente del espionaje), Toledo, Guadalajara, Teruel, Montalbán, Aragón, Almería y Valen-

${ }^{27}$ ENCINAS MORAL, Ángel L. (ed.): Las causas de la derrota de la República de España. Informe elaborado por Stoyán Mínev (Stepanov), Madrid: Miraguano, 2003, p. 226

${ }^{28}$ CDMH, INCORPORADOS, 737, Cp. 69, Exp. 10.

${ }^{29}$ SERRANO, S.: Maquis..., p. 44.

${ }^{30}$ Líster se quejará en sus memorias de que las condiciones para crear guerrillas no se aprovecharon por los diferentes Gobiernos republicanos y sus ministros de Defensa: LÍSTER, Enrique: Nuestra guerra, París, Editions de la Librairie du Globe, 1966, p. 277. José Díaz ante el Comité Central del P.C.E., reunido en Valencia del 5 al 8 de marzo de 1937, afirmó que "Hay que impulsar la formación de grupos guerrilleros que ya están actuando a espaldas la política criminal y de despojo de los fascistas". Vicente Uribe expresó que "la guerra podía acelerarse y tomar un rumbo victorioso por nuestra parte si sabíamos combinar la guerra regular con la irregular, es decir, si desencadenábamos la lucha armada y política en la retaguardia enemiga" y el propio Carlos García dijo que "Todo el gigantesco esfuerzo para organizar, armar y enviar al campo enemigo guerrillas se debía única y exclusivamente al Partido Comunista de España, el único decidido a ganar la guerra entre todos los partidos políticos del Frente Popular", en Archivo Histórico del Partido Comunista de España (en adelante AHPCE), Fondo tesis, manuscritos y memorias, Manuscritos 38/4, Memorias de Carlos García, ff. 4 y 6.

${ }^{31}$ PARSHINA, E.: La brigadista ..., p. 89.

${ }^{32}$ CDMH, INCORPORADOS, 737, Cp. 69, Exp. 5. 
$\operatorname{cia}^{33}$. Como puede deducirse fácilmente, los lugares elegidos eran limítrofes a las posiciones enemigas. Desde el campo propio se internaban con el propósito de llevar a cabo los siguientes objetivos:

1. Destrucción de los ferrocarriles y trenes con tropas y mercancías.

2. Cortar las comunicaciones telefónicas y telegráficas y destrucción de las fábricas eléctricas.

3. Hostilizar al enemigo en sus comunicaciones por carretera; destrucción de pequeños grupos enemigos durante su autotransporte. Capturar algunos coches con oficiales haciéndoles prisioneros.

4. Destrucción de los puentes más importantes para el transporte, por ferrocarril y carretera en el terreno enemigo.

5. Destruir fábricas de la industria militar, depósitos militares y depósitos de gasolina del enemigo.

6. Trabajo de destrucción en los puertos: provocar incendios en los depósitos de los mismos y en los barcos.

7. Ataque por sorpresa a los aeródromos, destrucción de los aeroplanos y personal de los mismos.

8. Organización del espionaje e información sobre el enemigo ${ }^{34}$.

Antes hemos hecho referencia al teniente coronel Santi o Ksanti. Este personaje no es otro que el agente soviético del NKVD, J.-U. Mámsurov, quien, "entre agosto de 1936 y octubre de 1937, dirigió todo el movimiento de guerrillas en España" y "sirvió de prototipo para el protagonista de la novela de Ernest Hemingway Por quién doblan las campanas: Robert Jordan, experto en subversión". ${ }^{35}$ En realidad Mámsurov ejerció más bien como líder o alma mater de estos grupos ya que su jefe oficial sería Alberto Calderón, y la dependencia orgánica de las guerrillas se le atribuyó desde un primer momento a la Sección de Información, dirigida entre marzo y julio de 1937 por el teniente coronel José Luis Coello de Portugal. Esta adscripción organizativa puede tal vez justificarse por la dificultad de encajar este tipo de guerra irregular dentro de las otras secciones de Estado Mayor ${ }^{36}$.

No en vano, al comienzo de esta regularización estas acciones fueron llevadas a cabo dentro de la Sección de Información por los denominados Grupos de Servicios Especiales (compañías de guerrilleros). Dichos Servicios Especiales actuaban como un cajón de sastre donde también se incluyeron los "inclasificables" espionaje y contraespionaje. Desde entonces, las guerrillas y sus acciones dependieron orgánicamente del Servicio de Información durante toda la guerra.

\footnotetext{
${ }^{33}$ Los cinco últimos (sin contar el de Teruel) se habían organizado recientemente.

${ }^{34} \mathrm{CDMH}$, INCORPORADOS, 737, Cp. 122, Exp. 4.

${ }^{35}$ RYBALKIN, Y.: Stalin..., p. 113.

${ }^{36}$ Las Secciones de Estado Mayor del Ejército eran cuatro: $1^{\mathrm{a}}$. Organización; $2^{\mathrm{a}}$. Información; $3^{\mathrm{a}}$. Operaciones; $4^{\text {a }}$. Servicios.
} 
La denominación de los grupos guerrilleros como Grupos de Servicios Especiales perduró hasta la primavera de 1937. En una carta remitida el 8 de abril por el jefe de la Segunda Sección al jefe de la Primera le advertía de que:

El Sr. Coronel Jefe de E.M. accidental, ha dispuesto que por esa Sección se estudie la organización de unidades de Información y servicios ofensivos, que con un carácter totalmente militar, y de acuerdo con la función que han de realizar en los distintos frentes, tengan una existencia absolutamente legal. [...] Estas unidades deberán constituirse a base de voluntarios [...] dependientes directamente de esta Sección de Información ${ }^{37}$.

Casi diez días después, el 17 de abril, estas renovadas unidades comenzaron a llamarse directamente Batallones de guerrilleros ${ }^{38}$. A partir de entonces, las guerrillas se organizaron, bajo la dirección de Alberto Calderón, en diez compañías de 125 hombres cada una, repartidas por diferentes frentes: $1^{\mathrm{a}}$. Belvis de la Jara (Toledo), $2^{\mathrm{a}}$. Don Benito (Badajoz), $3^{\mathrm{a}}$. Castuera (Badajoz), $4^{\mathrm{a}}$. Monterrubio de la Serena (Badajoz), $5^{\mathrm{a}}$. Villanueva de Córdoba (Córdoba), $6^{\mathrm{a}}$. Fuentes Calientes (Teruel), $7^{\mathrm{a}}$. Martos (Jaén), $8^{\mathrm{a}}$. Guadix (Granada), 9 ${ }^{\mathrm{a}}$. Cercedilla (Madrid) y 10 ${ }^{\mathrm{a}}$. Blesa (Teruel) ${ }^{39}$.

El hecho de que fuera aumentando el número de grupos guerrilleros puede tomarse como síntoma de su eficacia. Aunque algunas misiones tenían un objetivo algo prosaico, como aquella de marzo de 1937 en la que se ordenó a los guerrilleros que cruzasen las líneas enemigas para hacerse con un rebaño de $3.000 \operatorname{corderos}^{40}$, la mayoría de las infiltraciones tenían como objeto el sabotaje. La brigadista Elizaveta Parshina, perteneciente a la XI Brigada Internacional, relata en sus memorias algunos de estos actos llevados a cabo desde Málaga a Zaorejas (Guadalajara) pasando por el campo toledano. En ellos se procuraba la colocación de minas o dinamita en puntos clave como puentes o vías de ferrocarril. Otra muestra más de que estas acciones estaban dirigidas desde arriba lo confirma la dispensa de salvoconductos para cruzar las líneas por parte del propio Estado Mayor ${ }^{41}$.

Significativamente, el proceso de regularización y expansión se tornó por primera vez improductivo a finales de la primavera ya que "durante el mes de Mayo, solo dos de las seis compañías creadas se han distinguido por su actividad y fruto de en los trabajos emprendidos. Dichas Compañías, Tercera, con base en Castuera y Quinta, con base en Villanueva de Córdoba, han dado el resultado que se esperaba y calificamos su actuación como digna de elogio". Del resto se comentaba que habían tardado en organizarse (la $2^{\mathrm{a}}$, situada ahora en Villanueva de la Serena y la $6^{\mathrm{a}}$ que se había

\footnotetext{
${ }^{37}$ CDMH, INCORPORADOS, 737, Cp. 122, Exp. 4.

38 "Dependiente para su servicio peculiar de la Sección de Información del Estado Mayor del Ministerio de la Guerra se crea un 'Batallón de Guerrilleros'”, en CDMH, INCORPORADOS, 737, Cp. 122, Exp. 5.

${ }^{39}$ CDMH, INCORPORADOS, 737, Cp. 69, Exp. 6.

${ }^{40}$ PASTOR PETIT, D.: Espionaje (España 1936-1939), Bruguera, Barcelona, 1977, pp. 152-153

${ }^{41}$ PARSHINA, E.: La Brigadista..., p. 88.
} 
asentado finalmente en Cedrillas), que se les había puesto dificultades $\left(1^{\mathrm{a}}\right)$ o que su jefe era un irresponsable $\left(4^{\mathrm{a}}\right)^{42}$.

Poco tiempo duró el Batallón de Guerrilleros como unidad administrativa ya que el 7 de junio Indalecio Prieto firmó una orden disolviéndola, de tal manera que "[1] as Compañías ya creadas, y las que, en lo sucesivo, se creen, se afectarán, para su empleo y efectos administrativos, a los Cuarteles Generales del Ejército o Cuerpos de Ejército, en cuyo territorio operen". La mayoría se distribuyeron entre los Cuerpos de Ejército VII y VIII, que vivaqueaban en la zona extremeña, así como en la Agrupación Autónoma de Teruel.

Además, mediante dicha orden se pretendió crear otras cuatro compañías dentro de otras unidades en Guadalajara (Ejército de Centro), Jaén y Guadix (IX C.E.) y Sariñena (Ejército del Este). Por su parte, el jefe del Batallón de Guerrilleros, Alberto Calderón, siguió controlando en la práctica estas compañías ya que fue trasladado a la Sección de Información del E.M. del Ejército de Tierra "para la organización, instrucción e inspección de las Compañías de Guerrilleros, afectas a los Ejércitos"³.

\section{Composición, entrenamiento y algunas quejas (junio-septiembre 1937)}

Los jefes de estas guerrillas normalmente eran reclutados de entre los comisarios de las unidades regulares, mientras que los soldados, normalmente voluntarios, eran entrenados en una escuela situada en Benimamet (Valencia), dependiente de la Subsecretaría del Ejército de Tierra y cuyo carácter oficial fue otorgado por el Ministro de Defensa el mismo 7 de junio. La idea era formar un especie de elite de combate a través de esta escuela puesto que la mayoría de los individuos que cubrían las guerrillas hacia marzo de 1937 era más bien "vulgares reclutas de la última quinta: todos ellos de más de cuarenta años, 'amorosos' padres de familia, que pretextaban taras físicas reales o supuestas" ${ }^{\prime 4}$.

En la escuela se preparaba técnicamente a entre 20 y 25 alumnos de las distintas compañías con unos planes de estudio muy completos, aunque elementales, sobre aparatos, armamento, ametralladoras, pistolas automáticas, explosivos o electricidad. También había clases sobre la historia, los antecedentes y tipos de guerrilleros, completándose la educación con lecciones sobre instrucciones de asalto, burla a las precauciones enemigas o la elección del punto a atacar ${ }^{45}$.

Es muy posible que la creación de estas escuelas fuera sugerida por los asesores soviéticos. Para Daniel Kowalsky está claro que éstos "supervisaron en Barcelona y Valencia varios programas especiales de adiestramiento con el fin de preparar a francotiradores, colocadores de minas, expertos en el uso de ametralladoras, especialistas

${ }^{42}$ CDMH, INCORPORADOS, 737, Cp. 69, Exp. 6 (21 de junio de 1937).

${ }^{43}$ CDMH, INCORPORADOS, 736, Cp. 22, Exp. 1.

${ }^{44}$ AHPCE, Fondo tesis, manuscritos y memorias, Manuscritos 38/4, Memorias de Carlos García, f. 7. Esta imagen de soldados bastante mayores, e incluso de ancianos también en PARSHINA, E.: La Brigadista..., p. 37 .

${ }^{45} \mathrm{CDMH}$, INCORPORADOS, 736, Cp. 17, Exp. 23. 
en comunicaciones y personal capaz de reunir información para realizar trabajos en la retaguardia enemiga" ${ }^{46}$. Por su parte, el comunista Carlos García sostiene que durante el período de creación de una guerrilla "aparecieron breves días dos rusos que debían ser instructores o asesores", y también parece demostrado que los tres guerrilleros estadounidenses (los citados Goff, Aalto y Alex Kunslich) fueron entrenados en Albacete por instructores rusos ${ }^{47}$. Ahora bien, según las últimas investigaciones, el número de asesores rusos en esta materia tampoco fue demasiado elevado ${ }^{48}$.

Una vez entrenados, los guerrilleros eran dotados con un equipaje compuesto por "dos fusiles cada uno, dos dotaciones de balas, rancho en frío (conservas u chocolate), pastillas de trilita (parecidas a jabón de fregar), fulminantes, mecha rápida y lenta y propaganda. Los uniformes son los de campaña del extinto ejército español"49. En septiembre de 1937 los gastos mensuales derivados de la actividad de las compañías de guerrilleros ascendían a 20.000 pesetas, lo que equivalía a 2.000 pesetas por compañía. A esto debía añadírsele 511.700 pesetas distribuidas en concepto de pagas ${ }^{50}$. Para ese momento las compañías habían reubicado algunas de sus posiciones y contaban con un número menor de hombres de lo estipulado en teoría, aunque en todas ellas se llegaba a los cien individuos:

$1^{a}$ cía: Capitán Magín Molina Manzano en Talavera de la Reina; 110 hombres

$2^{a}$ cía: Capitán José Sines López en Don Benito; 112 hombres

$3^{\text {a }}$ cía: Capitán Miguel Julio Justo en Castuera; 112 hombres

$4^{\mathrm{a}}$ cía: Capitán Raimundo Bárcena en Villanueva de la Serena; 105 hombres

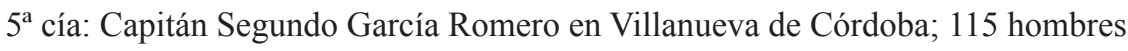

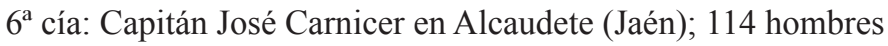

$7^{a}$ cía: Capitán Antonio Buitrago en Guadix (Granada); 100 hombres

$8^{\mathrm{a}}$ cía: Capitán Constantino Esteban en Alfambra (Teruel); 106 hombres

$9^{a}$ cía: Capitán Antonio Ojeda Ortega en Madrid (Navacerrada); 108 hombres

$10^{a}$ cía: Capitán Loubomif Tygy en Fiscal (Huesca-Pirineos); 100 hombres $^{51}$

${ }^{46}$ KOWALSKY, Daniel, La Unión Soviética y la guerra civil española, Barcelona, Crítica, 2004, p. 275; Aunque no aporta más pruebas que el hecho de que dos jefes guerrilleros -Luis Bárzana y Manuel Cristobal- fueran comunistas, Michael Alpert afirma que: "Parece pues muy probable que el XIV Cuerpo se hallase bajo una fuerte influencia comunista, lo que apunta aún más a actividades soviéticas" en ALPERT, M., El ejército..., p. 305.

${ }^{47}$ AHPCE, Fondo tesis, manuscritos y memorias, Manuscritos 38/4, Memorias de Carlos García. f. 7 y CARROLL, P. N: The Odyssey ..., p. 167.

${ }^{48}$ Se habla de siete personas para formar un grupo especial para actividades subversivas en un estadillo de septiembre de 1937 en VIÑAS, Ángel, El escudo de la República, Barcelona, Crítica, 2007, p. 238.

49 AHPCE, Fondo tesis, manuscritos y memorias, Manuscritos 38/4, Memorias de Carlos García.

${ }^{50}$ CDMH, INCORPORADOS, 737, Cp. 69, Exp. 11.

${ }^{51}$ CDMH, INCORPORADOS, 737, Cp. 69, Exp. 11. 
Sin embargo, a pesar de estas continuadas transformaciones y retoques, persistían en dicha compañías deficiencias que perjudicaban y retrasaban el trabajo guerrillero. El 18 de septiembre el Consejero del Grupo de Guerrilleros, que firmaba con el apodo de Víctor Hugo, dio a conocer los principales defectos de las mismas y las medidas de mejoramiento que debían plantearse ${ }^{52}$.

Entre las quejas llama la atención la mala calidad del armamento. Los guerrilleros portaban consigo fusiles y ametralladoras además de explosivos y provisiones. Un peso excesivo para aventurarse campo a través y que entorpecía su movilidad. De igual modo no existía un abastecimiento centralizado, lo que conllevaba que los guerrilleros no pudieran recibir los productos y materiales indispensables para el trabajo en la retaguardia del enemigo. Las provisiones, la ropa de abrigo y el tabaco escaseaban al cabo de unos días de trabajo en zona enemiga, lo cual afectaba también a la productividad de los guerrilleros. Destacan además otras carencias como pastillas de trilita, que obligaba a emplear dinamita, la cual era más peligrosa en su manejo; automóviles para un transporte rápido hasta las zonas de acción; medios para "la organización centralizada de la producción de alta calidad de los aparatos" y para el pago de los días, así como un reglamento de trabajo que especificara mejor las misiones guerrilleras y evitara el empleo de las mismas como tropas regulares.

En respuesta a estas demandas, se consideró la posibilidad de editar un reglamento, entregar armas más ligeras, centralizar y regular el abastecimiento, aumentar el parque automovilístico y designar un crédito de 5.000 pesetas al mes para cada compañía y otro también mensual de 15.000 para dotar mejor a la escuela, entre otras propuestas.

A estas alturas de la guerra, con el jefe de los guerrilleros clamando por una organización centralizada del servicio y tras haber sido acogidas en un primer momento bajo el manto de los Servicios Especiales de la Sección de Información, a Estrada no se le escapaba la posibilidad de aunar bajo su mando al total de las compañías de guerrilleros en el momento en el que estaba creando su Servicio de Inteligencia Militar. ${ }^{53}$ Por ello, desde septiembre de 1937 comenzó a enviar órdenes al Jefe del Batallón de Guerrilleros para que cada compañía remitiera informes desde sus respectivos sectores, comenzando así un proceso de unificación en torno a la Segunda Sección (Información) del Estado Mayor del Ejército de Tierra.

De esta época merecen destacarse los preparativos del ataque sobre Zaragoza, que terminaría derivando en la famosa batalla de Belchite. Según Rojo, "en la operación iba a participar además, irregularmente, un grupo de sesenta audaces guerrilleros que, infiltrados en la retaguardia enemiga, tenía la misión de ocupar los puentes de Zaragoza al amanecer de la segunda jornada, para impedir la organización de la resistencia enemiga en esos puntos sensibles o realizar, si era preciso, su destrucción",

\footnotetext{
${ }^{52}$ CDMH, INCORPORADOS, 736, Cp. 22, Exp. 1. Las siguientes líneas están sacadas de aquí.

${ }^{53}$ El Servicio de Inteligencia Militar no debe confundirse con el famosos S.I.M (Servicio de Investigación Militar). El primero fue creado en el verano de 1937 con el fin de centralizar y coordinar los distintos negociados dedicados a la obtención de información sobre el enemigo. Para un estudio monográfico del mismo vid. RODRÍGUEZ VELASCO, Hernán: Los Servicios de Información y Espionaje del Ejército Republicano en la Guerra Civil Española, Tesis Doctoral,Universidad de Salamanca, 2007.
} 
aunque finalmente el general Pozas, jefe de dicha ofensiva, rechazó la sugerencia de tomar Zaragoza al alba y por sorpresa utilizando a este grupo guerrillero ${ }^{54}$.

\section{Creación y desarrollo del SIAM o XIV Cuerpo de Ejército (octubre 1937- febrero 1938)}

Finalmente, un día de mediados de octubre se creó el Servicio de Información de Acción Militar (S.I.A.M.) que iba a ser dirigido por el inspector Domingo Ungría González y el mayor José Fernández Alabau ${ }^{55}$. Según el posterior Reglamento del Servicio de Inteligencia Militar, éste emplearía "los batallones o partidas de guerrilleros, teniendo por misión informar sobre la retaguardia enemiga, realizar en ella acciones de sabotaje y provocar y preparar, coadyuvando a él en su debido tiempo, el levantamiento en masa de la zona rebelde"56.

La creación del S.I.A.M bajo la tutela del Servicio de Información Militar contó con la complicidad del Jefe del Estado Mayor Central y del Ministro de Defensa, lo que vuelve a contradecir la idea de Stepanov de que "el movimiento guerrillero y el trabajo en la retaguardia del enemigo con Prieto permanecieron en el mismo estado embrionario en que también estaban con Caballero" 57 . De hecho, a partir de ese momento se crearon otras cuatro compañías de guerrilleros para cubrir los sectores de Barbastro, Cifuentes, Híjar y Almería ${ }^{58}$.

Lo que empezaba a crear fricciones entre los mandos era el control de los guerrilleros. Estrada defendía la dependencia orgánica de éstos del Servicio de Información alegando "el carácter secreto de las misiones" y una mayor coordinación en la retaguardia enemiga ${ }^{59}$. El mes de noviembre fue prolijo en la redacción de disposiciones para la organización en este sentido y para que las guerrillas empezaran a rendir cuentas de sus trabajos al propio Estrada ${ }^{60}$; y en diciembre, capeadas momentáneamente las dudas de Rojo, se dieron las primeras órdenes particulares para llevar a cabo unas misiones importantes ${ }^{61}$.

En efecto, en la jornada del 7 de diciembre se contó con los guerrilleros del S.I.A.M. para "evitar y cuando menos entorpecer o retrasar la afluencia de reservas enemigas a Teruel" durante los preparativos que se estaban haciendo para atacar dicha localidad. Los objetivos eran cortar las comunicaciones, hacer destrucciones,

${ }^{54}$ ROJO, Vicente: España heroica. Diez bocetos de la guerra española, Esplugues de Llobregat, Ariel, 1975, p. 108.

${ }^{55}$ No se conoce la fecha exacta. En ausencia de decretos de creación, el único criterio que tenemos para determinar ésta se debe a una documentación enviada por Estrada al jefe de los guerrilleros. El 11 de octubre esta correspondencia aún está dirigida a éste, pero seis días más tarde se remite al jefe del S.I.A.M. en CDMH, INCORPORADOS, 736, Cp. 4, Exp. 6.

${ }^{56} \mathrm{CDMH}$, INCORPORADOS, 675, Cp. 12, f. 25.

${ }^{57}$ ENCINAS MORAL: A. L. (ed.): Las causas..., p. 227.

${ }^{58} \mathrm{CDMH}$, INCORPORADOS, 736, Cp. 4, Exp. 8 (23 de octubre de 1937).

${ }^{59}$ CDMH, INCORPORADOS, 736, Cp. 22, Exp. 4 (28 de noviembre de 1937) .

${ }^{60}$ Ibidem.

${ }^{61} \mathrm{CDMH}$, INCORPORADOS, 736, Cp. 24. 
voladuras y sabotajes, al mismo tiempo que debían "informar sobre situación efectivos, identificación concentraciones y movimientos de las Unidades pertenecientes a las reservas enemigas que afluyan a Teruel" ${ }^{2}$. Del mismo modo, la unidad guerrillera formada por media docena de españoles, dos finlandeses y dos estadounidenses penetraron en territorio rebelde en la madrugada del 14 de diciembre para volar el puente que unía Teruel con Albarracín y cuyo éxito serviría posiblemente como modelo para la citada novela de Hemingway ${ }^{63}$.

Acabada la batalla de Teruel, el servicio de guerrilleros se reorganizó de nuevo con el visto bueno de Rojo y la aprobación final de Prieto. Se institucionalizaba, por fin, la acción del S.I.A.M. aunque en el borrador de dicho proyecto se predicaba que "para efectos de contra-espionaje y propaganda, en lo sucesivo las Unidades de Guerrilleros se denominarán XIV Cuerpo de Ejército" 64 . A partir de ese momento, las denominaciones de Cuerpo de Ejército XIV y S.I.A.M. se emplearon indistintamente para referirse a una misma realidad, aunque triunfó la primera opción.

En la cabecera del nuevo estatuto se reconocía oficialmente la actividad partisana y por ello se preveía un control más atento desde el E.M.C., aunque siempre a través de la Sección de Información de Estrada y el mando inmediato de Ungría como inspector jefe ${ }^{65}$. Con esta reorganización el Cuerpo de Ejército XIV pasaba a tener un total de 3.480 hombres, una cifra similar a los miembros de una Brigada Mixta. Se ampliaba a 24 el número de Unidades Guerrilleras, cada una de las cuales contaría con 145 guerrilleros, y se establecía en seis el número de Agrupaciones (580 guerrilleros), una por cada Ejército al que se afectaba. Este era su esquema final:
$1^{\text {a }}$ agrupación: División 48 (Brigadas 157, 158, 163, 230) Este
$2^{\mathrm{a}}$ agrupación: División 49 ${ }^{\mathrm{a}}$ (Brigadas 159,162, 165, 231) Levante
$3^{\mathrm{a}}$ agrupación: División $50^{\mathrm{a}}$ (Brigadas 155, 156, 164, 232) Centro
$4^{\mathrm{a}}$ agrupación: División 51 ${ }^{\mathrm{a}}$ (Brigadas 154, 160, 161, 233) Extremadura
$5^{\text {a }}$ agrupación: División $75^{\text {a }}$ (Brigadas 234, 235, 236, 237) Andalucía
$6^{\mathrm{a}}$ agrupación: División $76^{\mathrm{a}}$ (Brigadas 238, 239, 240, 241) Maniobra ${ }^{66}$

${ }^{62}$ Ibidem. En estas expediciones participaron los tres voluntarios estadounidenses según CARROLL, P. N.: The Odyssey..., pp. 167-168.

${ }^{63}$ Este episodio en LANDIS, Arthur: The Abraham Lincoln Brigade, New York, Citadel Press, 1967, p. 349 y CARROLL, P. N.: The Odyssey..., p. 167.

${ }^{64}$ CDMH, INCORPORADOS, 736, Cp. 22, Exp. 1 (3 febrero de 1938).

${ }^{65} \mathrm{CDMH}$, INCORPORADOS, 736, Cp. 74, Exp. 18.

${ }^{66}$ Hasta ese momento, el desarrollo del S.I.A.M. había sido muy lento. El 6 de noviembre se anunciaba que "El Sr. Ministro ha aprobado la creación del Cuerpo XIV con dos Divisiones y Cuartel General localizados en Parque Sama (Una División) y Valencia (Una División y Cuartel General), comunica a Inspectores Generales Servicios para que le doten de cuadros de mando y material. Por correo envío directivas y notas de autoridades a las que se han cursado" en CDMH, INCORPORADOS, 675, Cp. 11, f. 9; mientras que según Michael Alpert "En la lista de mando del 18 de diciembre de 1937 esta unidad [el XIV C.E.] aparece en proceso de organización, con tres números de División $\left(48^{\mathrm{a}}, 49^{\mathrm{a}}\right.$ y $50^{\mathrm{a}}$ )" en ALPERT, M., El ejército..., p. 271. 
De este modo, además, las viejas reivindicaciones parecían verse cumplidas: se preveía que "además de los devengos que por su categoría les corresponde, el personal de Jefes, Oficiales, Auxiliares y sargentos de las Unidades de Guerrilleros, percibirá una gratificación, por especialidad del servicio, de 200 pesetas mensuales; y los cabos y soldados, además de sus devengos correspondientes, otra gratificación especial de 6 pesetas diarias, conservando desde luego el derecho a las 2'25 pesetas diarias para alimentación" ${ }^{67}$. Incluso el presupuesto de Escuela de Guerrilleros que entonces era de 5.000 pesetas al mes, se vio aumentado hasta 30.000 pesetas mensuales para incluir en ella más alumnos y perfeccionar su técnica de enseñanza. Aparte se incluía un crédito mensual de 2.000 pesetas en moneda facciosa para la utilización en trabajos especiales por cada brigada ${ }^{68}$.

El cambio afectaba también a la relación de las guerrillas con los mandos de las diferentes unidades, ya que se disponía la colaboración entre ambos en aquellos frentes donde actuaran. Hasta ese momento la colaboración entre las tropas regulares y los grupos guerrilleros no había sido muy fluida. Sirva como ejemplo la situación dada el 28 de enero de 1938 en Alcaudete (Jaén) en la 76 Brigada Mixta. El jefe de ésta se quejaba de que un grupo de la $7^{\text {a }}$ Compañía de guerrilleros había salido al campo enemigo sin su autorización y además apuntaba que las actividades de éstos no eran "controlados por nadie dada la posición de insubordinación en que se han colocado los mencionados grupos; su estúpida manera de actuar les rebaja a la categoría de vulgares asaltadores de cortijos; quebranta el sigilo de los servicios especiales y cierra el camino de toda posibilidad de infiltración" ${ }^{69}$.

Con el nuevo reglamento se pretendía mejorar este tipo de asuntos. A partir de entonces los jefes de las guerrillas debían presentarse ante los jefes de las Divisiones o Cuerpos de Ejércitos y debían recibir de ellos auxilio, consejo, información sobre la zona y permiso para cruzar la línea enemiga Además, de los jefes de Ejército recibirían misiones operativas y debían comunicar a éstos sus resultados. Por último, esta reglamentación contemplaba cuidar de que otras guerrillas no legalizadas actuaran por su cuenta y riesgo ya que esto podía perturbar las acciones de otros servicios y además ofrecían un mal ejemplo en la retaguardia enemiga en la que se pretendía cambiar la situación ${ }^{70}$. Es decir, que a comienzos de 1938 seguían actuando guerrillas espontáneas, probablemente formadas por elementos de huidos que habían quedado aislados o por grupos anarquistas que aún se resistieron a la militarización forzosa. La CNT siempre se mostró favorable a este tipo de lucha, tan en la línea de su heterodoxa ideología, y precisamente hizo apología de ella en aquellos lugares donde los guerrilleros seguían luchando de manera irregular lejos de cualquier encuadramiento castrense, como en Huelva, Asturias o Galicia ${ }^{71}$.

\footnotetext{
${ }^{67}$ CDMH, INCORPORADOS, 736, Cp. 74, Exp. 18.

${ }^{68}$ Sin embargo, la cantidad total, unas 48.000 pesetas, no se había entregado aún en el mes de mayo. En una carta del propio Ministro de Hacienda a Estrada el 24 de ese mismo mes le comunicaba que no tenía disponible dicha cantidad. CDMH, INCORPORADOS, 736, Cp. 22, Exp. 1.

${ }^{69} \mathrm{CDMH}$, INCORPORADOS, 736, Cp. 18, Exp. 5.

${ }^{70} \mathrm{CDMH}$, INCORPORADOS, 736, Cp. 74, Exp. 18.

${ }^{71}$ ABAD DE SANTILLÁN, Diego, Por qué perdimos la guerra, Madrid, Ediciones G. del Toro,
} 


\section{Misiones, eficacia y problemas de mando (marzo-diciembre 1938)}

A diferencia de estas guerrillas espontáneas, las compañías guerrilleras ya reguladas debían cumplir ciertos objetivos previamente establecidos, tales como la interrupción de la circulación por tren y carretera mediante la "voladura de trenes y automóviles, puentes, túneles, carriles" o la "destrucción de objetivos militares y aniquilamiento de guardias enemiga, la interrupción de las comunicaciones telefónicas y telegráficas" y la recogida de "toda clase de información de la zona rebelde". En estas misiones los guerrilleros no debían rebasar los 50 kilómetros de profundidad y, en ningún caso debían ser utilizadas "como fuerzas regulares o de choque", algo que en ocasiones se olvidaba ${ }^{72}$.

Apenas dos meses más tarde de la reorganización de las guerrillas, el 27 de marzo, y como si la acción reguladora del Ministerio hubiera afectado a la labor de éstas, Ungría denunciaba el hecho de que "merma el prestigio y la eficacia de las Unidades del Cuerpo de Ejército XIV, una falta de trabajo casi absoluta" 73 .

Quizás como respuesta ante dicha crítica o como método para "mejorar, o al menos mantener, la moral republicana" dentro de la política de resistencia fijada por el Presidente Negrín ${ }^{74}$, el 23 de mayo se produjo el asalto al fuerte de Carchuna, cerca de Motril (Granada), en el que se liberó a más de 300 prisioneros republicanos encarcelados allí. La maniobra fue un espectacular golpe de mano nocturno dirigido por dos guerrilleros estadounidenses, Goff y Aalto, que "cortaron las líneas de comunicación. Mataron a los guardianes y armaron a los prisioneros con granadas". Tras la llegada de refuerzos enemigos, los americanos iniciaron una huida "de película": tras esconderse primero, se arrojaron al mar, donde permanecieron 48 horas, hasta que nadaron hacia la orilla enemiga, treparon por escarpadas rocas y llegaron finalmente a una granja ${ }^{75}$. Más allá de la increíble hazaña, lo más curioso de este episodio es que se realizó de manera espontánea sin consultar con el máximo responsable de las guerrillas, lo que demuestra que el espíritu de espontaneidad no había muerto del todo con la regulación. Estrada se quejaba al Jefe del XIV Cuerpo de que no había recibido "ningún parte ni siquiera indicación relativa a la acción desarrollada por los guerrilleros contra el fuerte de Carchuna en el sector de Motril. Es inadmisible que no tengamos ni de su proyecto ni de sus resultados otras noticias que las que ha dado la prensa y que ni siquiera se me haya dado conocimiento de una operación de su interés e importancia" $" 76$.

No sabemos si por coincidencia o porque aún le duraba el cabreo, sólo unos días más tarde Estrada volvía sobre Ungría, esta vez para reprocharle "la conducta obser-

pp. 235, 251-252. También en el artículo "Las guerrillas, modalidad de guerra popular que debe ser alentada", en Solidaridad Obrera, 27-5-1938 cit. en pastor petit, D.: Los Dossiers... pp. 147 y 151.

${ }^{72}$ CDMH, INCORPORADOS, 736, Cp. 18, Exp. 5.

${ }^{73}$ CDMH, INCORPORADOS, 736, Cp. 22, Exp. 13.

${ }^{74}$ GRAHAM, Helen: Breve historia de la Guerra Civil, Madrid, Espasa Calpe, 2006, p. 138.

${ }^{75}$ La descripción del asalto en LANDIS, A: The Abraham..., p. 490 y CARROLL, P. N.: The Odyssey..., p. 168.

${ }^{76}$ CDMH, INCORPORADOS, 736, Cp. 22, Exp. 4 (14 de junio de 1938). 
vada en el Cuartel General del Cuerpo de Ejército XIV, instalado en las inmediaciones de la Posición Pekín, en cuanto concierne al ambiente de francachela y diversión que en él se respira, y que contrasta con el duro trabajo que durante el día y la noche se observa en el Cuartel General del Grupo de Ejércitos"77.

La cacareada eficacia de las guerrillas parecía venirse al traste en cuanto se relegaba su espontaneidad inicial. Es posible que sus acciones perdieran el elemento sorpresa y la improvisación debido a que el enemigo le era ahora más fácil recabar los movimientos y organización de unos grupos cada vez más grandes e institucionalizados ${ }^{78}$.

Hasta julio de 1938 el XIV Cuerpo se había centrado hasta entonces en el sabotaje y la destrucción en la retaguardia enemiga ${ }^{79}$, pero a partir de entonces comenzó a apostarse por "explotar y alentar el estado de agitación, inquietud y revuelta que empieza a percibirse en el campo faccioso" y se propuso para ello "asignar a los guerrilleros la labor de preparar el levantamiento en masa de regiones como Extremadura y Andalucía, tan hostiles a la tiranía fascista" ${ }^{80}$. Se pretendía ampliar así la estrategia de desestabilización del frente interior enemigo, y parece que se logró al menos mantener un buen nivel de resultados durante el verano de 1938 a juzgar por tres hechos: la relación de felicitaciones recibidas, las informaciones de prensa extranjera que habían referido el trabajo de las guerrillas entre mayo y septiembre, y la intervención del Servicio de Información y Policía Militar (SIPM) del enemigo que ofrecía recompensas a la población civil para delatarlos y acabar así con sus acciones ${ }^{81}$.

Parece ser que esta medida de Franco consiguió reducir el trabajo de las guerrillas republicanas, que seguían inmersas, a finales de septiembre, en un largo proceso de reorganización. Para entonces, el total de fuerzas del XIV Cuerpo se fijaba en 3.480 soldados, unos 580 por división, aunque aún faltaban dos brigadas por crear (237 y 241) y "las restantes no están completamente con arreglo a la plantilla":

División 57ª (Brigadas 157, 158, 163, 230). Sector Andalucía Guadix (Granada)

División $200^{\mathrm{a}}$ (Brigadas 159, 162, 165, 231). Sector Extremadura Almadén

División $300^{a}$ (Brigadas 155, 156, 164, 232). Sector Centro Guadalajara

División 58 (Brigadas 154, 160, 161, 233). Sector Levante Betera

División $75^{\mathrm{a}}$ (Brigadas 234, 235, 236). Sector Este Guisona

División $76^{a}$ (Brigadas 238, 239, 240. Sector Ebro carretera a García a km y medio de Mora de Ebro $^{82}$.

\footnotetext{
${ }^{77}$ CDMH, INCORPORADOS, 736, Cp. 22, Exp. 4 (23 de junio de 1938).

${ }^{78}$ Esto no deja de ser una hipótesis propia sin ninguna base documental. Aún falta una monografía que evalúe la eficacia de las acciones guerrilleras.

${ }^{79}$ De hecho, a partir de mayo de 1938 se sucedieron una serie de órdenes desde el Servicio de Información para que el C.E. XIV llevara a cabo sucesivas misiones de sabotaje. La primera fue el 15 de mayo; la segunda el 10 de junio y la tercera el 15 de julio. En CDMH, INCORPORADOS, Caja 63, Cp. 3, Exp. 20.

${ }^{80} \mathrm{CDMH}$, INCORPORADOS, $675, \mathrm{Cp} .21$. proyecto de reorganización del Servicio de Inteligencia Militar

${ }^{81}$ Archivo Histórico Nacional, Archivo General Rojo, Caja 11/8 y SEIDMAN, Michael, A ras de suelo. Historia social de le República durante la Guerra Civil, Madrid, Alianza, 2006, p. 330.

${ }^{82} \mathrm{CDMH}$, INCORPORADOS, 736 , Cp. 22, Exp. 1 . El cambio de $200^{\mathrm{a}}$ por $49^{\mathrm{a}}$ y $300^{\mathrm{a}}$ por $50^{\mathrm{a}}$ lo aprobó Rojo el 23 de junio de 1938. (26 de septiembre de 1938).
} 
Sin embargo, lo que más preocupaba en el XIV Cuerpo era la batalla por su control. Hasta entonces las guerrillas habían dependido orgánicamente de la Sección de Información ${ }^{83}$, pero Rojo dispuso en octubre de 1938 su vinculación "a los Ejércitos, de cuyos jefes de Estado Mayor dependerán para toda clase de Servicios las Unidades que estén encuadradas en cada una de dichas Grandes Unidades" ${ }^{84}$. Esto no sentó nada bien a Estrada, quien siguió reclamando la dependencia para su Sección e incluso se manifestó a favor de duplicar el número de efectivos, que pasarían a 6.960 soldados, añadiendo además una plantilla de cabos (1.392) y un personal de telegrafistas $(32)^{85}$. Finalmente, tras varias negociaciones, Rojo y Estrada llegaron a un acuerdo por el cual las misiones clásicas podían ser asignadas por los jefes de E.M. de Ejércitos y Grupos de Ejércitos donde estuvieran encuadradas las unidades guerrilleras; pero otras misiones sólo podían ser fijadas exclusivamente por la Sección de Información del EM del Ejército de Tierra, como por ejemplo el ataque "a Puestos de Mando, parques, aeródromos", etcétera ${ }^{86}$.

El caso es que cuando se quiso solucionar definitivamente el tema sobre la tutela de las guerrillas, el tiempo se había echado encima y apenas faltaban unos meses para que concluyese la guerra. Y aunque "a principios de de 1939 se dio un gran aumento de las actividades guerrilleras en Extremadura"87, sus actuaciones poco pudieron hacer por revertir el trágico destino del bando republicano.

\section{Conclusiones}

El movimiento guerrillero republicano fue una realidad desde el comienzo del conflicto. Sin embargo, conviene distinguir entre las partidas de huidos del Norte de España que luchaban a la defensiva por su supervivencia y los guerrilleros de la zona Centro-Sur y Oriental que atacaban al enemigo para sabotear sus acciones. De estos últimos, implementados por el propio Estado Mayor del Ministerio de la Guerra, surgieron los grupos que lucharían posteriormente como unidades reguladas dentro del Ejército Popular de la República. De hecho, la mayoría de los guerrilleros que combatieron en la Guerra Civil lo hicieron encuadrados en las filas del Ejército, para quienes cumplían sus misiones, salvo los huidos que no pudieron conectar con el territorio leal o aquellos que no quisieron ser militarizados.

Ha quedado demostrado, por vez primera con documentación republicana, que la inserción de estas guerrillas en dicho Ejército fue un largo proceso que comenzó oficialmente en diciembre de 1936, aunque desde agosto se permitieran ya los golpes de mano a las milicias regulares e irregulares en zonas porosas de Aragón o Extremadura. Durante

${ }^{83}$ Algo que ya reseña Parshina en sus memorias: “Oficialmente José y su destacamento dependían del jefe de la segunda sección del Estado Mayor de la XI Brigada Internacional” en PARSHINA, E.: La Brigadista..., p. 288.

${ }^{84}$ Orden Circular, artículo 24 en CDMH, INCORPORADOS, 674, Cp. 10, f. 24 (1 de octubre 1938).

${ }^{85}$ CDMH, INCORPORADOS, 736, Cp. 22, Exp. 1 (2 de octubre de 1938).

${ }^{86} \mathrm{CDMH}$, INCORPORADOS, 736, Cp. 22, Exp. 1 (12 de noviembre de 1938).

${ }^{87}$ ALPERT, M., El Ejército..., p. 272. 
meses, los partisanos fueron organizándose en grupos y unidades dependientes de la Sección de Información. Aunque las diferentes reestructuraciones y cambios en la dirección durasen prácticamente hasta finales de 1938, la creación del S.I.A.M. o XIV Cuerpo de Ejército en octubre de 1937 significó el momento de mayor madurez organizativa. Este Cuerpo de Guerrilleros, pertenecía orgánicamente a la Segunda Sección del Estado Mayor, puesto que era parte de un todo más grande, el Servicio de Inteligencia Militar, y una de sus misiones era la recogida de información. En el plano operativo, sin embargo, sus misiones eran asignadas por los Estados Mayores de las unidades en las que formaban parte.

La relación entre guerrilleros y soldados regulares no fue siempre buena y las tensiones entre ambos grupos de hombres a veces crearon disputas dentro de las unidades. Los partisanos se sabían una "raza" distinta al resto de sus compañeros: habían sido formados específicamente en escuelas con instructores soviéticos, cobraban más y sus labores de infiltración y sabotaje les permitían evitar la confrontación directa con el enemigo. A cambio, sus misiones eran más arriesgadas y peligrosas.

Frente a la idea tradicional de que los mandos superiores nunca confiaron en el desarrollo de las guerrillas, hemos demostrado que los militares republicanos José Eduardo Villalba, Vicente Rojo y Manuel Estrada fueron los pioneros en el fomento de estos grupos irregulares. Asimismo, los Ministros de Defensa Largo Caballero, Indalecio Prieto y Juan Negrín secundaron también su utilización, aunque fueron los anarquistas, por razones obvias, y el Partido Comunista con los consejeros soviéticos quienes más apostaran por el empleo de las guerrillas.

Otra cosa es pensar que un uso más extendido de éstas hubiera supuesto un final de la guerra alternativo, aunque la mayoría de los estudiosos han descartando la eficacia de una lucha basada exclusivamente en la "guerra irregular" ${ }^{88}$. Lo único cierto es que, una vez acabada la guerra, algunos guerrilleros republicanos continuaron combatiendo junto a la Resistencia francesa durante la Segunda Guerra Mundial, siendo "un cuerpo armado autónomo, dedicado a realizar sabotajes, atacar destacamentos alemanes y colaborar en las redes de evasión" $"$. Por su parte, los que se quedaron en España fueron reagrupándose en partidas cada vez más grandes y organizadas que lograron resistir casi dos décadas al régimen franquista, aunque en este caso no hubo continuidad con la lucha ejercida por el XIV Cuerpo ${ }^{90}$. Ambos grupos aplicaron, eso sí, las enseñanzas aprendidas durante la Guerra Civil Española. Los resultados, sin embargo, fueron dispares: unos vencieron al fascismo en Europa y fueron reconocidos internacionalmente, mientras que los otros perdieron y su memoria quedó en el olvido hasta hace poco tiempo.

${ }^{88}$ CARDONA, Gabriel: "Entre la revolución y la disciplina. Ensayo sobre la dimensión militar de la guerra civil", en MORADIELLOS, Enrique (ed.), La Guerra Civil, Ayer, no 50 (2003), p. 42; SEIDMAN, M.: A ras..., p. 122. Para Abraham Guillén, sin embargo, el error fatal de la República fue crear un ejército regular en vez de continuar la revolución popular con la lucha de guerrillas y del pueblo: GUILLÉN, Abraham, El error militar de las «izquierdas». Estrategia de la guerra revolucionaria, Barcelona, Hacer, 1980.

${ }^{89}$ BEEVOR, Antony: La Guerra Civil Española, Barcelona, Crítica, 2005, pp. 661-662.

${ }^{90}$ HEINE, Hartmut: "El Partido Comunista Español y la organización del fenómeno guerrillero", en ARÓSTEGUI, J. y MARCO, J. (eds.): El último..., p. 82. 